\title{
Analysis of the Advantages and Disadvantages of Android and iOS Systems and Converting Applications from Android to iOS Platform and Vice Versa
}

\author{
Lazarela Lazareska, Kire Jakimoski \\ Faculty of Informatics, FON University, Skopje, Republic of Macedonia
}

Email address:

lazarela.lazareska@fon.mk (L. Lazareska),kire.jakimoski@fon.edu.mk (K. Jakimoski)

To cite this article:

Lazarela Lazareska, Kire Jakimoski. Analysis of the Advantages and Disadvantages of Android and iOS Systems and Converting Applications from Android to iOS Platform and Vice Versa. International Journal of Engineering Management.

Vol. 6, No. 5, 2017, pp. 116-120. doi: 10.11648/j.ajsea.20170605.11

Received: September 12, 2017; Accepted: September 23, 2017; Published: October 28, 2017

\begin{abstract}
This paper strives for a better understanding of Android and iOS mobile operating systems, their history and overall development to today, a detailed research of Android and iOS performance, determining their strengths and flaws, and their comparative analysis. The goal is to provide a detailed study of the strengths and weaknesses of Android and iOS platforms and converting applications from Android to iOS platform and vice versa with the aim to increase their usage in the mobile application market, therefore the time and costs needed for development and realization to be significantly reduced, in order to provide application content designed by the Android platform to be usable on the iOS platform and vice versa.
\end{abstract}

Keywords: Analysis, Android, Application, Automatic Conversion, Convertor, iOS, Operating System, Programming

\section{Introduction}

Today there is a number of mobile operating systems that have tried to be the best in the world, but none has managed to climb to the top and make such impact as iOS and Android. Android is a software set of software subsystems needed to provide a fully functional solution for mobile devices. $\mathrm{iOS}$ is a mobile operating system that allows all other applications to run on one of its iPhone, iPod Touch or iPad products. Both platforms contain similarities in method of use and the devices that are used, but there are many differences that result from different operating systems: Android and iOS. These challenges are thoroughly explored and explained in this paper.

In the mobile phone market, the emergence of various smartphone platforms such as Android and iOS forcing developers to constantly develop applications with the same content in order to ensure compliance with each of the available platforms. Then, each application developed from one platform in order to be used on another platform must be reprogrammed. As a result, it takes considerable time and cost to analyze and convert the contents of a mobile application to use a platform on a smart phone.

By automatically converting existing application content to the content of other application content, existing application content can quickly be downloaded to different platforms. As a result, their usage will increase, and therefore the workforce, time and costs will be reduced.

In this paper, in order to solve this problem, automatic converting and converters designed to automatically translate Android content into iOS and vice versa has been studied.

\section{Android Operating System and Play Store}

Android is a mobile operating system developed by Google, which is primarily intended for mobile devices such as smartphones. The operating system uses touch inputs such as dragging, tapping and pinching to manipulate display objects and virtual keyboard. In 2015, Android had the largest installed base on all mobile operating systems.

In June 2017, the Google Play Mobile App Store has released over 3,000,000 Android apps, and over 80 billion applications have been downloaded. In 2017, the Google I / O company discovered that they had over 2 billion active Android users per month, unlike the previous year, with a figure of approximately 1.5 billion active users. 
Starting from 2008 to the present, Android has had many upgrades that have been gradually improved its operating system by adding new features and fixing errors in previous versions. Each new version is named after a dessert in alphabetical order: Cupcake 1.5; Donut 1.6; Eclair 2.0; Froyo 2.2; Gingerbread 2.3; Honeycomb 3.0; Ice Cream Sandwich 4.0; Jelly Bean 4.1; KitKat 4.4; Lollipop 5.0; Marshmallow 6.0; Nougat 7.0 and the latest Oreo 8.0 version [1] [2] [3].

\section{3. iOS Operating System and App Store}

iPhone operating system or iOS is a mobile operating system developed in 2007 by Apple Inc., which works exclusively on Apple's mobile devices such as iPhone, iPad, iPod touch, Apple TV and other Apple devices.

By January 2017, Apple's App Store contains more than 2.2 million iOS applications. All these mobile applications were downloaded more than 130 billion times. In 2017 the operating system had 710 million active devices. In the latest data obtained on the basis of the reports received in the third quarter of 2017, Apple announced that it has sold more than 1.2 billion iOS devices.

Apple Inc. almost every year provides updates to the iOS operating system for iPhone, iPad and iPod touch via iTunes, and from iOS 5.0 version provides over-the-air software updates. All available versions of iPhone are: iPhone OS 1, iPhone iOS 2, iPhone iOS 3, iOS 4, iOS 5, iOS 6, iOS 7, iOS 8, iOS 9, iOS 10 and the latest iOS 11 version [6] [7] [8].

\section{Analysis of the Advantages and Disadvantages of Android and iOS Platforms}

Regarding the analysis made in various categories, I can conclude that in the interface section iOS is convincingly leading over Android because of its more uniform, stylish and convenient interface, while in terms of user experience slight advantage is given to Android due to the huge set of choosing menus, their ease of use and an ideal customer QWERTY keyboard [9].

In the application availability section, iOS runs over Android due to the simpler interface, higher buying of expensive games, and thus more profitability and more recommendations for new applications [10] [14].

Following the operating system stability section, and the research conducted in the first quarter of 2017, it was found that $68 \%$ of iOS devices experienced a failure concerning the number of problems, application decline, overheating of the device and drop in connectivity, etc., while for Android devices this figure is $50 \%$ [19]. In the research, the results showed that Android applications were more likely to fall by $18 \%$, while the decline in iOS applications was $50 \%$. As the most unstable iOS models are mentioned iPhone 6, iPhone 6s, iPhone $5 \mathrm{~s}$ and iPhone 6 Plus, while Samsung, Xiaomi, Motorola, Sony and Lenovo are mentioned as the most uns Android manufacturers. In terms of the most unstable apps on the iOS operating system are Facebook, Instagram, Google,
Facebook Messenger, Snapchat, and Pinterest appear on Android, while on the Android system are IMS Service, Facebook, Goggle Play Services, TouchWiz Home and Address Book. As the final conclusion about the stability of the systems, it can be said that Android is more serious and stable system in terms of the iOS system [11] [12] [13].

Regarding software upgrades, both platforms are regularly and timely updated and there is a lot of control over their software. However, preference is given to Apple because it offers a safer and more up-to-date update of its versions, while on Android depending on the mobile operator of the user who is testing the new versions depends when the new updates are going to be provided [20].

It's hard to compare these two platforms when it comes to battery life due to the diversity of their hardware. iPhone is limited with size of its battery, while Android devices have various sizes of batteries that can easily overpower the iPhone. Apple's battery is still unchangeable, unlike Android that has a variable battery and thus increases the utilization of the Android device. Newer models of Android, with the exception of the standard wired battery charging, also include wireless charging, which is slower than the standard yet gives great convenience to users, while in Apple models this feature is still unavailable. In this section, Android is in great advantage because of its greater benefits over the iPhone [21].

In the camera's device area, a small advantage is given to iOS because it extracts the maximum values from its hardware. By comparing the 12 megapixel camera on the iPhone 7 and the 16 megapixel camera of the LG G5, the iPhone has far more powerful camera than just a megapixel. The camera and platform applications themselves are fast and easy to use, although the iPhone's preference is given for greater ease of use and better image results without filtering them.

The analysis made in terms of peripheral devices with Android and iOS preference is given to iOS because Apple has built a larger ecosystem of peripherals (iPad, Apple Watch, MacBook and HomeKit) and in that way has a larger number of peripherals devices.

In terms of routing, launching the operating system and jailbreak, Android is the winner because of the possibility of routing the mobile device. With the routing mobile device has access to multiple applications and is available for the latest system updates, greater aesthetic enhancements to the operating system, increased device speed, increased battery life and many more. While iOS has jailbreak feature of the device that allows downloading and installing apps outside the App Store and removing some restrictions, it's still not as effective as Android routing [16] [22].

With Android and iOS settings, there are many different elements, but a huge advantage is given to Android because the user can customize his experience in many ways, there is a possibility to install various launchers to change the look of the user interface, a possibility to set up more backgrounds in the background and shortcuts and also opportunity to choose its default keyboard, while iOS 8 supports third-party keyboard, but it's not even close to that of Android [24].

The connectivity on both platforms is classical because it 
supports Bluetooth and Wi-Fi. Android uses NFC, i.e. wireless communication in a short field, while iOS has this feature included on the iPhone 6 and $6+$. In this category, Android wins because NFC is unlocked and it is much easier to add third party add-ons [23].

When choosing devices, Android is incredibly leading due to the huge variety of various mobile devices, while iOS is only available on Apple devices.

The basic functionality for calling and messaging is effective on both platforms. Android offers Hangouts, Messenger, Allo and Duo, through which users can send messages over the Internet or SMS, while iOS has FaceTime and iMessage that offer many more features, GIFs, stickers, integration of third-party applications and according to stated and according to ease of use, iOS wins. Regarding standard email applications, both platforms use a number of third-party email applications. However, the Gmail app on Android is more popular and more powerful than the e-mail application from iOS Mail and it offers the user an option to add more email addresses from various providers. Because of this reason, the advantage is given to Android [29].

Each platform uses its own web browser, Google Chrome on Android and Safari on iOS. Both browsers are high quality with the difference that Chrome is available for iOS, while Safari is not for Android. Both, Android and iOS offer good maps and accurate estimates, but the quality of Google Maps is unbeatable. It contains more points and is more detailed compared to Apple Maps. Safety on both platforms is at a satisfactory level. Due to the huge focus on rapid updates, Android lags behind with the security of its system compared to the iOS system. A number of Android devices use software that is older than 3 years and are therefore susceptible to serious hacking that is a threat to security. Apple is working to improve its security with the Touch ID that represents fingerprint recognition. Increased surveillance over iOS applications and the ability to update multiple devices more quickly takes advantage over system security [17] [18] [31].

The privacy of iOS users is on high level because user personal data is encrypted and not read, used, or stored by Apple, while Android is less encrypted and privacy is more unprotected. That's why Apple offers the best protection for the privacy of its users.

As a platform iOS is simpler and easier to use because it is the same in all iOS devices, unlike Android that is different on each device from different manufacturers. Also iOS is less cumbersome and more fashionable than most Android phones and it makes iOS a winner in this category.

And finally, in terms of creating and publishing applications, Android and Play Store are winning because of their greater freedom and cheaper registration fee [30].

\section{Study of Converting Applications from Android to iOS Platform and Vice Versa}

An analysis of a mobile application has been made that automatically converts Android apps into iOS and iOS apps in Android with the help of content analyzer, resource converter, engine mapping engine, and source translator. The Android platform developed by Google is an optimized platform for mobile devices with the perfect combination of operating system, middleware and application programs. The iOS platform developed by Apple is the world's most advanced mobile operating system, which is constantly redefining its devices. By converting Android apps into iOS, resources such as images and sounds can be converted, the API can be converted using the platform mapping engine, and the application sources can be converted using a source translator with compiler-writing technology [26] [27].

The analyzer analyzes the input content and produces output where the resource data and source code stored in the content are separated. A resource converter is a system that converts text or binary data from an application that needs to be converted to image, sound, and user data, so that it can be used for the purpose of the platform of the file system. The engine mapping platform is a system that provides API functions that allow the previous platform to recreate the executable environment using the target platform functions. The platform can supports the functions to work in the same form and also it enables the converted codes to be easily understood and changed [28].

It also takes care of the reliability and stability of performance from the homogeneity of the environment. The source translator receives Android codes that are produced by the content analyzer and translates them into iOS source codes that are semantically equivalent and perform the same function on the Android platform.

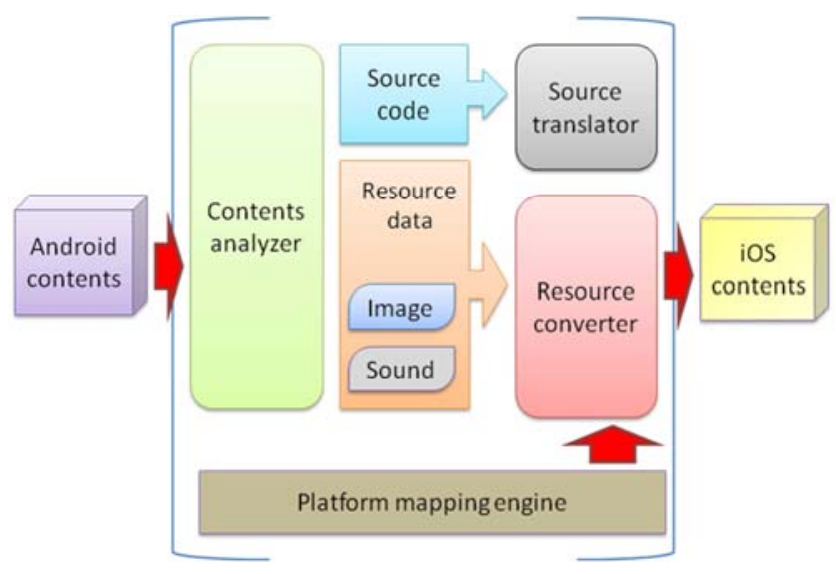

Figure 1. Model on Android - iOS converter.

\section{Programming Android and iOS Application}

An environment for studying the conversion of Android and iOS applications has been developed using the Xamarin tool to create mobile applications with their natural interface. The new application consists a music player and a picture gallery. It has been tested and functional on both platforms, Android and iOS. 

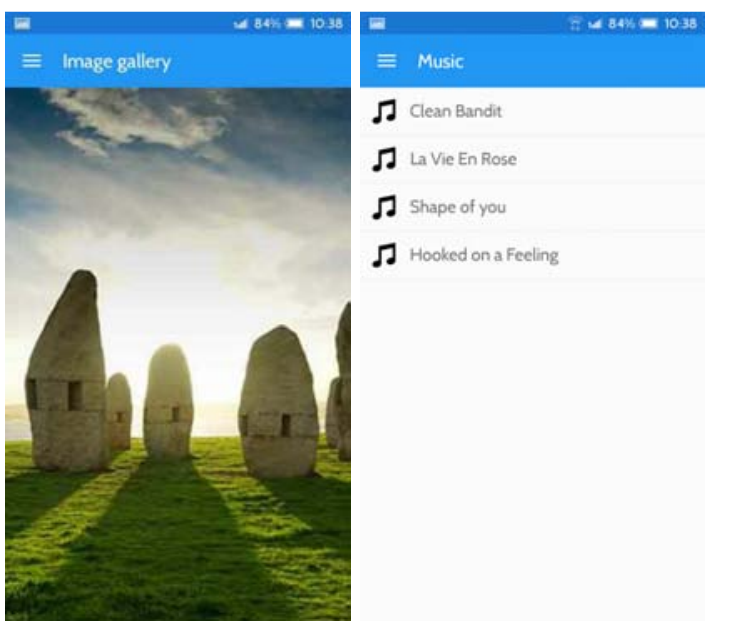

Figure 2. Display of a Part of Android Application.

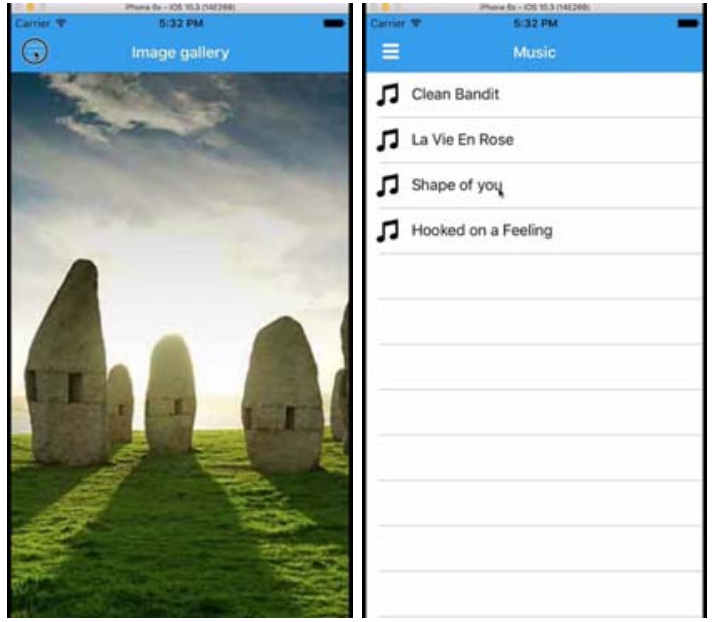

Figure 3. Display of a Part of iOS Application.

There are no differences between the two programming languages, because Xamarin uses the C\# programming language to create both applications. Regarding the design, Android has more precision, clarity and naturalness, while the iOS design is more undefined, contains larger images, negative space and the most commented difference among users is that there is no button "Back". In terms of application programming, Android application programming meant application programming that would be used on a large number of Android devices, with different screen sizes and various ratios, while iOS is standard for its devices. For these reasons, there is fragmentation of Android versions, and because of that, the programming of Android apps is very complicated, unlike iOS where the situation is convincingly easier and it saves a lot of time for developers [4] [5] [15] [25].

\section{Conclusion}

Through the analysis of both platforms Android and iOS as a winner of this "battle", Android comes out with a very small difference of 9 vs. 8. Although Android is a winner, iOS is a more favorable operating system because of its victories in design, safety, upgrade, applications and ecosystem categories. iOS guarantees timely software updates, prioritizes the privacy of users, has a better ecosystem of applications and games compared to Android. For these reasons, as an overall winner, I declare Apple and its iOS.

The final results obtained in converting applications showed that the image, audio signal, graphics and other features of the converted iOS application were the same as those of the Android app and vice versa. With automatic converting of content, it can be transferred to different platforms. As a result, the utilization of the application will increase, the time and costs will be reduced.

\section{References}

[1] Google Inc. (2017) Android, Available on: https://www.android.com/

[2] Wikipedia (2017) Android operating system, Available on: http://en.wikipedia.org/wiki/Android_\%28operating_system\% 29

[3] “Google, Android”, http://code.google.com/intl/ko/android/

[4] R. Schwarz, P. Dutson, J. Steele and N. To, "The Android Developer's Cookbook: Building Applications with the Android SDK”, Addison-Wesley Professional, (2013).

[5] P. Deitel, H. Deitel, D. MacLean, “Android: How to Program”, Prentice Hall, (2014).

[6] Apple Inc. (2017) iOS, Available on: https://www.apple.com/ios/

[7] Wikipedia (2017) iOS, Available on: http://en.wikipedia.org/wiki/IOS

[8] "Apple, iOS Technology Overview", $\mathrm{http}: / /$ developer.apple.com/devcenter/ios

[9] Brad Yale. (2014). Operating System Battleground: iOS vs. Android. Available on:

http://www.informit.com/blogs/blog.aspx?uk=Operating-Syst em-Battleground-iOS-vs-Android.

[10] David Nield (2015) iOS vs Android: The 2015 Edition, Available on:

http://fieldguide.gizmodo.com/ios-vs-android-the-2015-editio n-1700461435

[11] Derek Walter (2015) Apple iOS 8 v Android 5.1 - which is best?, Available on:

http://thenextweb.com/dd/2015/04/06/apple-ios8-v-android-51-which-is-best/

[12] David Nield (2015) Android vs iOS: What's the Best Mobile OS in 2015?, Available on:

http://www.lifehacker.co.uk/2015/04/29/android-vs-ios-whatsthe-best-mobile-os-in-2015.

[13] Scientiamobile (2017) Mobile Overview Report January March 2017, Scientiamobile.

[14] Neil Aitken (2015) Android vs iOS vs Windows Phone 8 Mobile OS Comparison, Available on: http://whatphone.com.au/compare/android-vs-ios/ 
[15] Mark Oldytowski (2015) Android vs. iOS from a Developer's Perspective, Available on:

$\mathrm{http}: / /$ sphereoi.com/studios/android_vs ios/

[16] Keir Thomas (2015) Android Lollipop vs iOS 8 comparison review, Available on:

http://www.macworld.co.uk/review/ios-apps/apple-ios-8-vs-g oogle-android-lollipop-review-3529028/

[17] Google report (2015) Android Security 2014 Year in Review, Google.

[18] Apple (2015) iOS Security iOS 8.3 or later, Apple.

[19] Wikipedia (2017) Comparison of mobile operating systems, Available on http://en.wikipedia.org/wiki/Comparison_of_mobile_operatin g_systems

[20] Good Technology (2015) Mobility Index Report Q1 2015, Good Technology.

[21] Jillian D'Onfro (2015) 15 Reasons Android Phones Are Better Than iPhones, Available on:

http://www.businessinsider.com/android-phones-versus-iphon e-2015-1?op=1

[22] Florence Ion (2014) 10 Android features that still make it better than iOS 8, Available on:

http://www.greenbot.com/article/2686006/10-android-features -that-still-make-it-still-better-than-ios-8.html

[23] Alan Lu (2014) Android Kit Kat 4.4 vs Apple iOS 7 head-to-head review, Available on: http://www.itpro.co.uk/mobile/21021/android-kitkat-44-vs-ap ple-ios-7-head-head-review/page/0/2
[24] Linda Federico-O'Murchu (2015) Why people pick Team Apple versus Team Android, Available on: http://www.cnbc.com/id/102289985

[25] Xamarin. 2017. Building Cross Platform Applications. [ONLINE] Available at: https://developer.xamarin.com/guides/cross-platform/applicati on_fundamentals/building_cross_platform_applications/

[26] Y. S. Lee, "Automatic Mobile Contents Converter for Smart Phone Platforms", Journal f Korea Multimedia Society, vol. 15, no. 54, (2011).

[27] Y. S. Lee, J. S. Kim and M. J. Kim, "Development of the Contents Analyzer and the Resource Converter for Automatic Mobile Contents Converter", Journal of Korea Multimedia society, vol. 14, no. 681, (2011).

[28] J. H. Kim and Y. S. Lee, "A Study on the iOS-to-Android Converter using a Resource converter and a Platform Mapping Engine", International Journal of Software Engineering and its Applications, SERSC, vol. 8, no. 427, (2014).

[29] Aijaz A. Sheikh, Prince T. Ganai, Nisar A. Malik "Smartphone: Android Vs IOS", The Standard International Journals (The SIJ). vol. 1, no. 4, (2013).

[30] Shivam Jaiswal, Ajay Kumar "Research on Android app Vs Apple app Market: Who is leading?", International Journal Of Engineering And Computer Science, vol 3 Issue 4, (2014) Page No. 5553-5556.

[31] Yogita Chittoria, Neha Aggarwal "Application Security in Android-OS VS IOS?", International Journal of Advanced Research in Computer Science and Software Engineering, vol 4, Issue 5, (2014). 